\title{
STRUCTURAL MODIFICATION OF ESCHERICHIA COLI PEPTIDOGLYCAN INDUCED BY BICYCLOMYCIN
}

\author{
A. G. Pisabarro, F. J. Cañada, D. Vázquez*, \\ P. Arriaga and A. Rodríguez-TÉbar \\ Instituto de Biología Molecular, Consejo Superior de Investigaciones Científicas, \\ Centro de Biología Molecular, Universidad Autónoma, \\ E-28049-Madrid, Spain
}

(Received for publication December 30, 1985)

\begin{abstract}
We have studied the modification of Escherichia coli peptidoglycan induced by bicyclomycin. For this purpose liquid chromatography for peptidoglycan analysis has been used. The main alteration found was an increase of diaminopimelyl-diaminopimelyl bridge containing subunits. Our results show that bicyclomycin impairs the normal breakage of that interpeptidic bond, whose cleavage is needed for the normal remodeling of peptidoglycan and cell growth. Based on the analysis of the possible structure of diaminopimelyl-diaminopimelyl bond and bicyclomycin, we propose a hypothesis on the mechanism of action of bicyclomycin.
\end{abstract}

Bicyclomycin is an antibiotic which was first isolated from Streptomyces sapporonensis ATCC 21532, by the Research Laboratories of Fujisawa Pharmaceutical Co., Ltd. ${ }^{1}$. This antibiotic is active against a number of Gram-negative bacteria, but ineffective against Gram-positive microorganisms²). TANAKA et al..$^{3)}$ reported that bicyclomycin inhibited protein synthesis and particularly synthesis of envelope proteins, mainly lipoprotein. These authors found that the levels of murein-bound lipoprotein in bicyclomycin-treated Escherichia coli cells, were decreased. Morphological studies carried out by SOMEYA et al. $\left.{ }^{4}\right)$ have shown that bicyclomycin induces the formation of blebs on the cell surface as well as a highly undulated outer membrane. However, later work by HiRotA et al..$^{5)}$ demonstrated that mutants lacking lipoprotein could grow normally under a variety of conditions. These data indicated that, at least, the main killing action of bicyclomycin is not at the lipoprotein level ${ }^{8}$. The existence of seven minority proteins located in the cytoplasmic membrane of E. coli that bound ${ }^{14} \mathrm{C}$-bicyclomycin was later shown ${ }^{6)}$. These bicyclomycin-binding proteins (BBPs) were shown to be distinct from penicillin-binding proteins (PBPs) previously described by SPRATT ${ }^{7)}$. Nevertheless, despite those findings, the mechanism of action of bicyclomycin remains unknown although some reasonable hypotheses have been made with regard to the mode of action of the antibiotic with its target proteins ${ }^{8 \sim 10)}$. In this paper we report the structural modifications of E. coli peptidoglycan induced by bicyclomycin. From our data, we put forward a hypothesis on the mode of action of the antibiotic.

\section{Materials and Methods}

\section{Bacterial Strains and Growth Conditions}

E. coli ATCC 27166 (a mutant hypersensitive to bicyclomycin ${ }^{2)}$ and E. coli W7 (dapA, lys A) were used in this study. Both strains were grown in $\mathrm{L}$ medium supplemented with $4 \mathrm{~g} /$ liter of $\mathrm{D}$-glucose and, only for W7 strain, $5 \mathrm{mg} /$ liter of 2,6-meso-diaminopimelic acid. Cells were grown under forced aeration and established conditions (see the legend of Fig. 1 and results) and were collected by rapid cooling and centrifugation. 
Fig. 1. Action of bicyclomycin on the growth of E. coli ATCC 27166 (A) and E. coli W7 (B). Upward arrows: addition of bicyclomycin at concentrations $(\mu \mathrm{g} / \mathrm{ml})$ for A: (-) 0 , (O) 4, (A) 8 ,

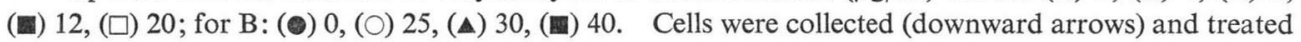
for peptidoglycan analysis. In the case of W7 strain, cells had been labeled with $\left[{ }^{3} \mathrm{H}\right]-2,6-$ meso-diaminopimelic acid 15 minutes before harvesting.
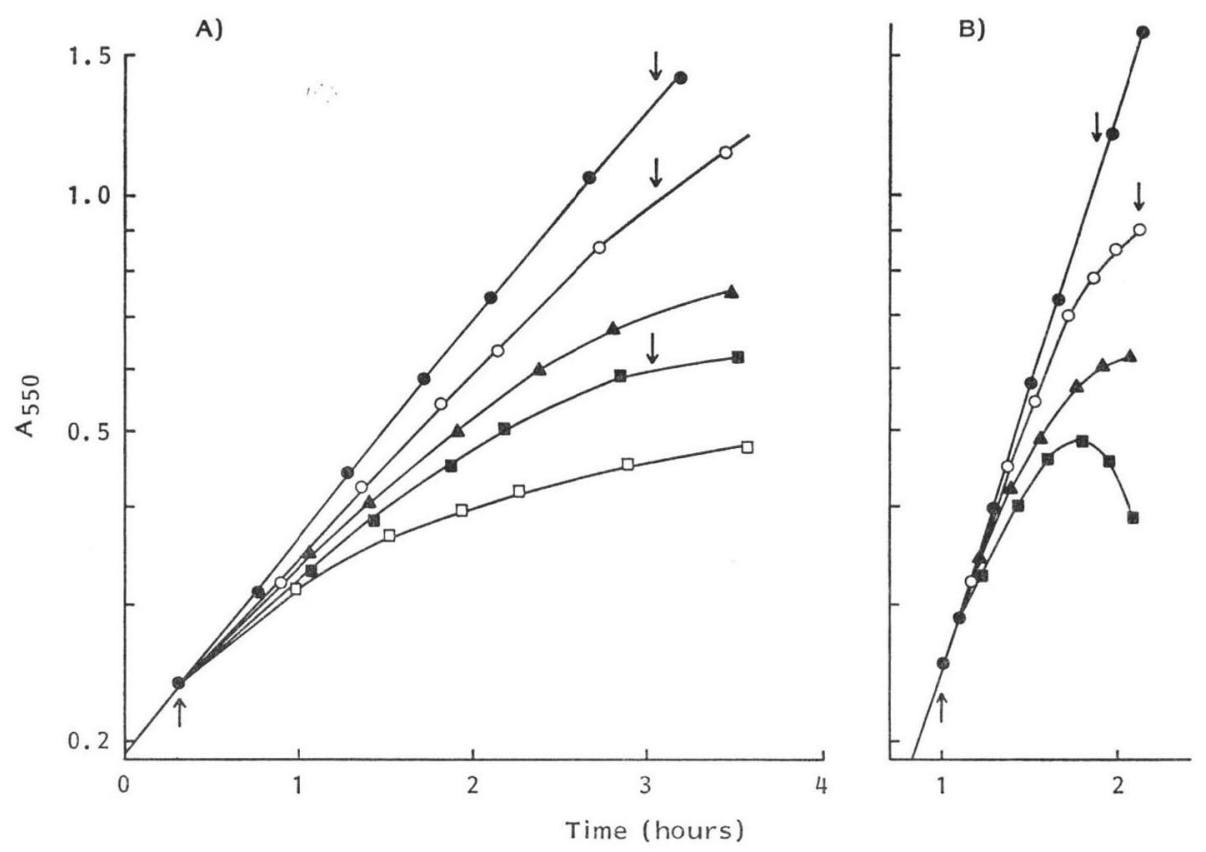

Radioactive Labeling of E. coli Peptidoglycan

E. coli $\mathrm{W} 7$ cells growing exponentially in a culture were treated with $\left[{ }^{3} \mathrm{H}\right]-2,6-m e s o$-diaminopimelic acid (Commisariat à l'Energie Atomique, Gif-sur-Yvette, France) at a final concentration of $5 \mu \mathrm{Ci} /$ $\mathrm{ml}$. The addition of the radioactive precursor was done as illustrated in Fig. 1. The incubation was continued for 15 minutes and after that cells were collected as described above.

Isolation and Structural Analysis of Peptidoglycan

Centrifuged cells were treated for the isolation of peptidoglycan as described previously ${ }^{11}$. This procedure includes the treatment of peptidoglycan by pronase which cleaves off most bound lipoprotein polypeptide, leaving only a lysyl-arginine residue attached to peptidoglycan. Deproteinized peptidoglycan was hydrolyzed by "Chalaropsis" muramidase as described ${ }^{12)}$. Peptidoglycan subunits were resolved by high pressure liquid chromatography (HPLC). The procedure for peptidoglycan fractionation by HPLC as well as the structural analysis and identification of chromatogram peaks have been reported previously ${ }^{13)}$.

\section{Results}

Fig. 1A shows the effects of several concentrations of bicyclomycin on E. coli ATCC 27166. The antibiotic, at the concentrations used did not produced cell lysis although all concentrations caused a clear inhibition of the growth rate. Examination of bicyclomycin-treated cells, under light microscopy (not shown) revealed that the antibiotic induced the formation of filaments as described before ${ }^{2}$. Cells were collected as indicated by the downward arrows of Fig. 1A and, subsequently, peptidoglycan was isolated and prepared for HPLC analysis. The molar fraction of each individual subunit from either bicyclomycin-treated or bicyclomycin-untreated cells is shown in Table 1. In Table 2, the dif- 
Table 1. Composition of peptidoglycan from E. coli ATCC 27166 cells treated with bicyclomycin ${ }^{a}$.

\begin{tabular}{|c|c|c|c|}
\hline \multirow{2}{*}{ Peptidoglycan subunits ${ }^{b}$} & \multicolumn{3}{|c|}{ Bicyclomycin $(\mu \mathrm{g} / \mathrm{ml})$} \\
\hline & 0 & 4 & 12 \\
\hline 1. Tri & 10.66 & 11.66 & 22.04 \\
\hline 2. Tetra $(\text { Gly })^{\mathrm{c}}$ & 1.32 & 1.46 & 0.81 \\
\hline 3. Tetra & 50.67 & 48.90 & 36.65 \\
\hline 4. Penta $(\mathrm{Gly})^{\mathrm{c}}$ & ND & ND & ND \\
\hline 5. Dipeptide & 3.03 & 3.31 & 4.06 \\
\hline 6. Penta & 1.05 & 0.80 & 0.41 \\
\hline 7. Tri-Lys-Arg ${ }^{\mathrm{d}}$ & 5.40 & 4.77 & 3.79 \\
\hline 8. Tetra-tri $(\text { Gly })^{\mathrm{c}}$ & 0.09 & 0.11 & 0.41 \\
\hline 9. Tri-tri(DAP-DAP) ${ }^{\mathrm{e}}$ & 0.18 & 0.22 & 0.81 \\
\hline 10. Tetra-tri & 1.25 & 1.39 & 2.10 \\
\hline 11. Tetra-tri(DAP-DAP) ${ }^{\ominus}$ & 1.32 & 1.86 & 3.79 \\
\hline 12. Tetra-tetra $(\mathrm{Gly})^{\mathrm{c}}$ & 0.66 & 0.86 & 1.89 \\
\hline 13. $\operatorname{Tri}(\operatorname{anh})^{\mathrm{f}}$ & ND & ND & ND \\
\hline 14. Tetra-penta $(\text { Gly })^{c}$ & 0.20 & 0.20 & 0.20 \\
\hline 15. Tetra-tetra & 19.21 & 18.09 & 15.01 \\
\hline 16. Tetra-penta & 0.13 & 0.33 & 0.20 \\
\hline 17. Tri-tri(DAP-DAP)-Lys-Arg ${ }^{\mathrm{d}, \mathrm{e}}$ & 0.07 & 0.13 & 0.14 \\
\hline 18. Tetra $(a n h)^{\mathrm{f}}$ & 0.13 & 0.27 & 0.14 \\
\hline 19. Tetra-tetra-tri(DAP-DAP) $^{\mathrm{e}}$ & 0.13 & 0.22 & 0.72 \\
\hline 20. Tetra-tetra-tetra & 1.01 & 0.93 & 1.40 \\
\hline 21. Tetra-tri(DAP-DAP)-Lys-Arg ${ }^{\mathrm{d}, \mathrm{e}}$ & 0.92 & 1.39 & 2.23 \\
\hline 22. Tri-tri(DAP-DAP) $(\mathrm{anh})^{\mathrm{e}, \mathrm{f}}$ & ND & ND & ND \\
\hline 23. Tetra-tri-tri(DAP-DAP)-Lys-Arg ${ }^{\mathrm{d}, \mathrm{e}}$ & 0.18 & 0.18 & 0.14 \\
\hline 24. Tetra-tri $(a n h)^{\mathrm{f}}$ & 0.66 & 0.73 & 0.20 \\
\hline 25. Tetra-tri(DAP-DAP) $(\mathrm{anh})^{\mathrm{e}, \mathrm{f}}$ & 0.20 & 0.27 & 0.74 \\
\hline 26. Tetra-tetra $(a n h)^{\mathrm{f}}$ & 0.92 & 0.99 & 1.35 \\
\hline 27. Tetra-tetra-tri $(a n h)^{\mathrm{f}}$ & ND & 0.04 & ND \\
\hline 28. Tetra-tetra-tri(DAP-DAP) $(a n h)^{e, f}$ & ND & 0.09 & 0.14 \\
\hline 29. Tetra-tetra-tetra $(\operatorname{anh})^{\mathrm{f}}$ & 0.31 & 0.35 & 0.45 \\
\hline 30. Tri-tri(DAP-DAP)-Lys-Arg $(a n h)^{\mathrm{d}, \mathrm{e}, \mathrm{f}}$ & 0.33 & 0.46 & 0.20 \\
\hline
\end{tabular}

a The table indicates the molar fraction $\times 100$ of each mucopeptide in each condition.

b In this nomenclature the disaccharide part is ommitted for the purpose of clarity. The prefixes di-, tri-, tetra- and penta- mean disaccharide di-, tri-, tetra- and pentapeptide respectively.

c (Gly) means that the last amino acid of the peptide is glycine substituting to D-alanine.

d Lys-Arg means lysyl-arginine residue which comes from lipoprotein which was bound to the subunit before treating peptidoglycan with pronase.

e (DAP-DAP) means the existence of a diaminopimelyl-diaminopimelyl interpeptide bridge.

f (anh) means anhydro sugar subunit which constitutes the terminal component of a glycan chain. The ratio non-anhydro/anhydro subunits gives the length of the glycan chain.

ND: Not detected.

ferent subunits of Table 1 have been grouped into families. From Tables 1 and 2, the following conclusions should be pointed out: i) There was a noticeable increase of disaccharide tripeptide (Table 1, No. 1); ii) the amount of disaccharide tetrapeptide was reduced (Table 1, No. 3); iii) the amount of bis-disaccharide tetrapeptide was also decreased (Table 1, No. 15); iv) disaccharide tripeptide subunits containing lipoprotein were greatly reduced (Table 1, No. 7). However, v) the total amount of disaccharide peptides, bis-disaccharide peptides and tri-disaccharide peptides, was scarcely affected by bicyclomycin (Table 2, Nos. 1, 2 and 3). Consequently, vi) the degree of interpeptide cross-linking of peptidoglycan from bicyclomycin-treated cells changed very little (Table 2, No. 4). This degree of 
Table 2. Composition of peptidoglycan from E. coli ATCC 27166 cells treated with bicyclomycin. Data are from Table 1 but here the individual subunits have been grouped into families ${ }^{a}$.

\begin{tabular}{llrr}
\hline & & \multicolumn{3}{c}{ Bicyclomycin $(\mu \mathrm{g} / \mathrm{ml})$} \\
\cline { 2 - 4 } Subunit family & \multicolumn{1}{c}{0} & \multicolumn{1}{c}{4} \\
\hline 1. Monomers $^{\mathrm{b}}$ & 72.3 & 71.2 & 68.0 \\
2. Dimers $^{\mathrm{b}}$ & 26.1 & 27.0 & 29.2 \\
3. Trimers $^{\mathrm{b}}$ & 1.6 & 1.8 & 2.8 \\
4. Bridges $^{\mathrm{c}}$ & 29.4 & 30.7 & 35.0 \\
5. DAP-DAP & 3.3 & 4.8 & 8.9 \\
6. DAP-DAP/Bridges & 11.3 & 15.7 & 25.5 \\
7. Lipoprotein $^{\mathrm{e}}$ & 6.9 & 6.9 & 6.5 \\
8. Lipoprotein D-T $^{\mathrm{f}}$ & 21.7 & 31.2 & 41.7 \\
9. Anhydro compounds & 2.5 & 3.2 & 3.2 \\
10. Glycan chain length & & 31.2 & 31.2 \\
\hline
\end{tabular}

a The table indicates the molar fraction $\times 100$ of each family in each condition.

b Total amount of monomers (disaccaride peptides), dimers (bis-(disaccharide peptides)) and trimers (tri(disaccharide peptides)).

c Total amount of dimers plus trimers, i.e., those subunits containing interpeptide bridges.

d Total amount of dimers and trimers which have one diaminopimelyl-diaminopimelyl interpeptide bridge.

e Total amount of subunits containing one terminal lysyl-arginine residue derived from hydrolyzed lipoprotein.

f Percentage of cross-linked subunits (dimers and trimers) containing lipoprotein $(100 \%=$ total subunits containing lipoprotein).

$\mathrm{g}$ Anhydro compounds are the terminal subunits of a glycan chain. The ratio non-anhydro/anhydro compounds estimates the chain length, i.e., the number of disaccharide subunits per chain.

cross-linking was not maintained by the usual interpeptide D-alanyl-diaminopimelyl bridges whose amounts actually decreased (see, e.g., Table 1, No. 15); but vii) rather was maintained by the striking increase of cross-linked subunits containing interpeptide diaminopimelyl-diaminopimelyl (DAP-DAP) bonds (Table 2, No. 5 and Table 1, Nos. 9, 11, 17, 19, 21, 25 and 28). In fact, viii) the ratio between the two kinds of bridges, i.e., DAP-DAP versus D-alanyl-diaminopimelyl, increased considerably, the former bridges representing above $25 \%$ of the total bridges (Table 2, No. 6).

Fig. 1B, shows the effect of bicyclomycin on the growth of E. coli W7 cells. It can be seen that this strain is not as sensitive to bicyclomycin as is the ATCC 27166 strain. Indeed, concentrations of bicyclomycin up to $20 \mu \mathrm{g} / \mathrm{ml}$ (not shown) did not produce any noticeable effect on cell growth. However, concentrations above $25 \mu \mathrm{g} / \mathrm{ml}$ produced retardation of the growth and eventual cell lysis. Microscopic examination (not shown) revealed that bicyclomycin induced morphological changes in W7 cells. Such alterations mainly consisted of shortening of the rods to form round or spheroplast-like cells, which exploded with time, but we failed to detect any sort of filaments in bicyclomycin-treated W7 cultures. However these cells were collected for peptidoglycan analysis when they were still growing at a nearly normal rate.

Table 3 shows the primary structure of peptidoglycan from both bicyclomycin-treated or untreated W7 cells. Changes in mature peptidoglycan (lanes A and B) are not large, but, there are significant differences between untreated and bicyclomycin-treated cells. Indeed, the amount of subunits containing interpeptide DAP-DAP bridges decreased upon antibiotic treatment (Table 3, Nos. 5 and 6). Furthermore, the amount of lipoprotein bound to the peptidoglycan also decreased (Table 3, No. 7), although the percentage of lipoprotein bound to dimers and trimers increased (Table 3, No. 8). Nevertheless, possibly a finer picture of bicyclomycin-induced alteration on peptidoglycan, emerges 
Table 3. Composition of peptidoglycan from E. coli W7 cells either untreated (A, C) or treated with 25 $\mu \mathrm{g} / \mathrm{ml}$ bicyclomycin (B, D).

A and $B$ represent the molar fraction $\times 100$ of subunit groups from total cell peptidoglycan while $\mathrm{C}$ and $\mathrm{D}$ show the molar fraction $\times 100$ of subunit groups from de novo peptidoglycan labeled with

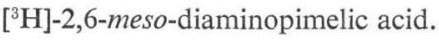

\begin{tabular}{|c|c|c|c|c|}
\hline Subunit family* & A & B & $\mathrm{C}$ & $\mathrm{D}$ \\
\hline 1. Monomers & 70.6 & 71.0 & 75.7 & 74.8 \\
\hline 2. Dimers & 28.1 & 28.2 & 23.8 & 24.5 \\
\hline 3. Trimers & 1.3 & 0.8 & 0.4 & 0.7 \\
\hline 4. Bridges & 30.7 & 29.7 & 24.7 & 25.9 \\
\hline 5. DAP-DAP & 4.5 & 3.8 & 3.8 & 5.1 \\
\hline 6. DAP-DAP/Bridges & 14.7 & 12.9 & 15.5 & 19.6 \\
\hline 7. Lipoprotein & 7.4 & 4.7 & 5.0 & 3.1 \\
\hline 8. Lipoprotein D-T & 26.1 & 39.4 & 38.3 & 57.6 \\
\hline 9. Anhydro compounds & 2.1 & 1.3 & 1.2 & 1.5 \\
\hline 10. Glycan chain length & 48.0 & 74.0 & 82.3 & 66.4 \\
\hline
\end{tabular}

* See notes of Table 2 .

from pulse-labeling experiments (lanes $\mathrm{C}$ and $\mathrm{D}$ ). As can be observed, the most noticeable change is that de novo peptidoglycan from bicyclomycin-treated cells contained an increased amount of subunits containing the DAP-DAP interpeptide linkage (Table 3, Nos. 5 and 6). The amount of bound lipoprotein also decreased although, again, the percentage of lipoprotein bound to cross-linked subunits was increased by bicyclomycin treatment (Table 3, Nos. 7 and 8).

\section{Discussion}

Cell Alterations Induced by Bicyclomycin

It had been reported previously ${ }^{2)}$ that bicyclomycin produced mixed effects on the morphology of $E$. coli cells, i.e., the antibiotic produced both round-shaped or spheroplast-like cells and filaments. In this study we have chosen two $E$. coli strains which responded differently to bicyclomycin. Under our growth conditions the antibiotic exclusively produced filaments in the ATCC 27166 strain, while exclusively round-shaped cells were seen in the W7 strain. These distinct effects suggest, at first glance, that bicyclomycin might act at different levels, and some of them could predominate in one and others in another strain. Concomitantly, the effects produced by bicyclomycin on the peptidoglycan structure from either ATCC 27166 or W7 cells could differ from each other. The finding that bicyclomycin specifically binds to a few proteins of the cytoplasmic membrane of $E$. coli ${ }^{6)}$ prompted us to initiate this study especially since new technology for peptidoglycan analysis has been developed ${ }^{13)}$. Our results ndicate that bicyclomycin induces two main kinds of alterations in peptidoglycan. First, the antibiotic increases the amount of DAP-DAP bridges in peptidoglycan. As demonstrated by GLAUNER and SCHWARZ $^{13)}$, DAP-DAP bridges are very rapidly produced by nascent peptidoglycan. Also lipoprotein very rapidly gets bound to, and only to, DAP-DAP containing subunits. Later, the amount of DAP-DAP containing subunits, either with or without lipoprotein decreases, while there is an increase of disaccharide tripeptide containing lipoprotein, obviously because the DAP-DAP linkage is hydrolyzed. From our data, it seems that bicyclomycin impairs to a great extent the hydrolysis of DAP-DAP bonds. As a result, the amount of DAP-DAP containing subunits increases. Furthermore, our data show that the level of lipoprotein bound to cross-linked subunits is higher in antibiotictreated cells while the amounts of disaccharide tripeptide carrying lipoprotein are greatly reduced, the later could not be generated from lipoprotein bound cross-linked subunits.

The second effect, induced by bicyclomycin on cell peptidoglycan, is of more diffuse characteristics. The amount of cross-linked species containing the more usual D-alanyl-diaminopimelyl interpeptide bridges decreases. On the other hand, the amount of disaccharide-tripeptide is also increased upon 
Fig. 2. A) Structure of bicyclomycin assessed by X-ray crystallography analysis (refs 18 and 19).

B) Proposed structure of the DAP-DAP linkage.

A)

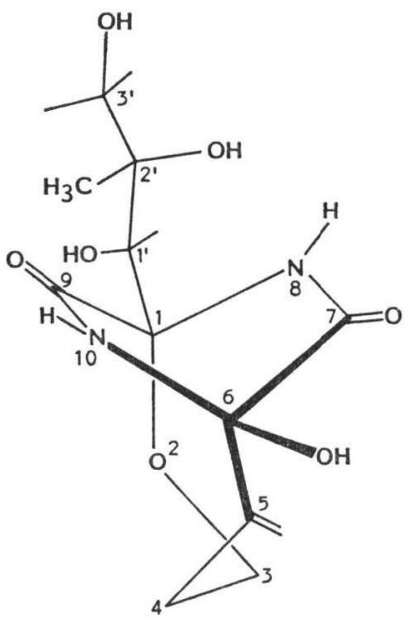

B)

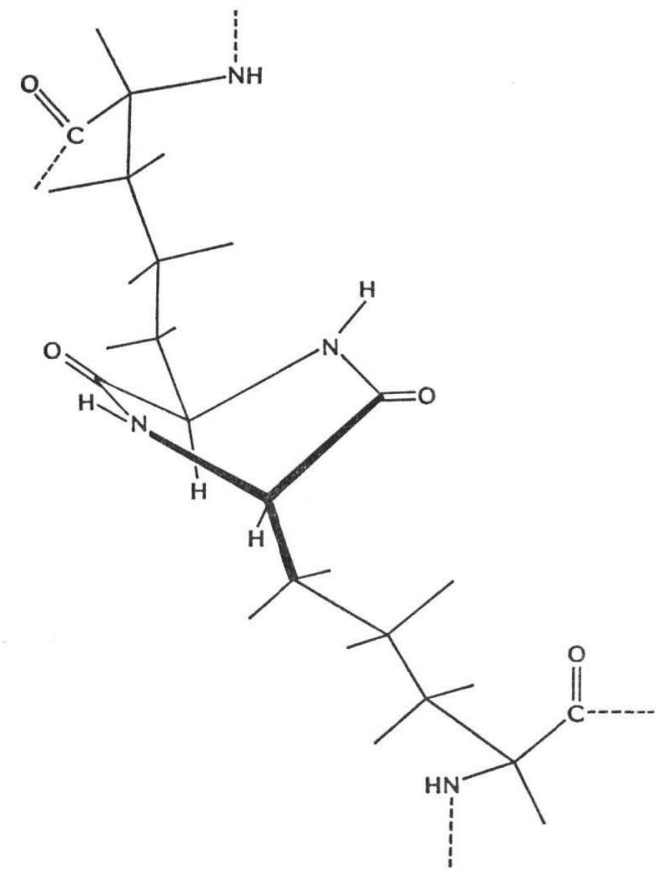

bicyclomycin treatment. These effects could be due to some difficulty in obtaining the usual crosslinking from de novo pentapeptide. Non utilized pentapeptide excess could undergo degradation to tetra and tripeptide by the action of DD and LD carboxypeptidases.

\section{The DAP-DAP Bond}

The existence of di- and trimeric subunits, whose interpeptide bridge is a DAP-DAP bond, was discovered by Glauner and SchwARZ ${ }^{13)}$. This is a peptidic (amide) bond as it is broken by acid hydrolysis. In normal $E$. coli cells, DAP-DAP bonds are responsible for around $12 \sim 17 \%$ of the total interpeptide cross-linking of peptidoglycan. The relative amount of DAP-DAP bonds increases up to $25 \%$ in the stationary phase of cell growth ${ }^{14,15)}$. However, many questions concerning the structure of this bond and the source of energy for its formation, remain to be answered. Certainly, this bond involves the two D centers of meso-diaminopimelic residues because of the existence of the tetratri subunit containing both a DAP-DAP bond and lipoprotein which is linked to the $\mathrm{L}$ center of the diaminopimelic residue ${ }^{16)}$. The formation of the first amide bond involving one $-\mathrm{COOH}$ and one $-\mathrm{NH}_{2}$ groups brings about the formation of a second amide bond which is energetically favored. The final result could be a six member-ring lactam (diketopiperazine ring, see Fig. 2A).

The Mechanism of Action of Bicyclomycin. An Hypothesis

The targets of bicyclomycin in the bacterial membrane (BBPs) have not yet been characterized. Therefore, nothing is known about the structure of the antibiotic-enzyme complex. In order to ascertain the place of binding of bicyclomycin in the target enzyme, and the transformations undergone by the antibiotic molecule, studies on the reactivity of bicyclomycin for nucleophilic reagents have been carried out. In a first study, SOMEYA et al. ${ }^{8)}$ inferred that the active center of bicyclomycin could be the terminal olefinic group that can undergo a nucleophilic attack by thiols forming an adduct whose structure was determined. These authors concluded that the target protein could be a sulfhydrylenzyme that might exert a similar nucleophilic attack on bicyclomycin. Later, MüLler et al. ${ }^{17)}$ synthesized a large number of bicyclomycin derivatives. Some compounds (e.g. No. 57 of ref 15) had the terminal olefinic group substituted by an oxime and still retained their biological activity. Reaction of 
thiols with the oxime group would be less favored. More recent studies by WiLLIAMS and his co-workers showed a lack of correlation between simple thiolate susceptibility and biological activity of bicyclomycin. This indicates that this interesting reaction alone cannot be used as the biomechanistic template $^{9)}$. These authors in another article ${ }^{10)}$ proposed a very interesting mechanism of action of bicyclomycin. The receptor would be a protease-like enzyme which performs a nucleophilic attack on the ketone 9-carbon of the diketopiperazine ring of the antibiotic. Thus, the 9, 10-amide bond would be cleaved and an acyl-enzyme intermediate formed. We propose that the mechanism of action of bicyclomycin is mainly due to the analogy of the diketopiperazine ring of this antibiotic with the lactam involved in the DAP-DAP bonds (see Fig. 2). The four member bridge between positions 1 and 6 in the molecule of bicyclomycin would maintain the diketopiperazine ring in a forced boat conformation, which is a structural analog of the DAP-DAP bond. Thus bicyclomycin would inactivate the amidase enzyme responsible for the breakage of the DAP-DAP bond. As a result DAP-DAP cross-linked subunits would increase in cell peptidoglycan, as shown by our biochemical data. The excess of DAPDAP linkage and the difficulty of their breaking would impede, on the one hand, the normal growth of the cell, and, on the other hand, these bonds could impair the formation of the more usual diaminopimelyl-D-alanine interpeptide bonds from nascent pentapeptide, as reflected in our biochemical data. Such impairment could be derived from steric factors that would impede the remodeling of growing peptidoglycan whose structure would remain blocked by uncleavable DAP-DAP bonds.

According to this hypothesis, the lack of sensitivity of Gram-positive bacteria to bicyclomycin could be explained by the fact that most of these bacteria lack $\gamma$-meso-diaminopimelic acid which impairs the formation of DAP-DAP bond and, in addition, Gram-positive bacteria do not have lipoprotein or outer membrane.

\section{Acknowledgments}

Bicyclomycin was a generous gift from Fujisawa Pharmaceutical Co., Ltd. A.G.P. and F.J.C. are holders of fellowships from the Fondo de Investigaciones Sanitarias and the Juan March Foundation respectively. This work has been supported by grants No. 661-512 and 603-416 of the Caicyt and by an institutional grant of the Fondo de Investigaciones Sanitarias de la Seguridad Social.

\section{References}

1) Mryoshi, T.; N. Mryairy, H. Aoki, M. Kohsaka, H. Sakai \& H. Imanaka: Bicyclomycin, a new antibiotic. I. Taxonomy, isolation and characterization. J. Antibiotics 25: 569 575, 1972

2) Nishida, M.; Y. Mine, T. Matsubara, S. Goto \& S. Kuwahara: Bicyclomycin, a new antibiotic. III. In vitro and in vivo antimicrobial activity. J. Antibiotics 25: 582 593, 1972

3) Tanaka, N.; M. Iseki, T. Mryoshi, H. Aoki \& H. Imanaka: Mechanism of action of bicyclomycin. J. Antibiotics 29: 155 168, 1976

4) Someya, A.; K. Tanaka \& N. TAnaka: Morphological changes of Escherichia coli induced by bicyclomycin. Antimicrob. Agents Chemother. 16: 87 91, 1979

5) Hirota, Y.; H. Suzuki, I. Nishimura \& S. Yasuda: On the process of cellular division in Escherichia coli: A mutant lacking a murein lipoprotein. Proc. Natl. Acad. Sci. U.S.A. 74: 1417 1420, 1977

6) Someya, A.; M. Iseki \& N. TANaKA: Binding of bicyclomycin to inner membrane proteins of $E$. coli. J. Antibiotics 31: 712 718, 1978

7) Spratt, B. G.: Properties of the penicillin binding proteins of Escherichia coli K-12. Eur. J. Biochem. 72: $341 \sim 352,1977$

8) Someya, A.; M. Iseki \& N. TAnakA: Active groups of bicyclomycin and the reaction with thiols. J. Antibiotics 32: 402 407, 1979

9) Williams, R. M.; K. Tomizawa, R. W. Armstrong \& J.-S. Dung: Thiolate additions to bicyclomycin and analogues: A structurally novel latent Michael-acceptor system. J. Am. Chem. Soc. 107: 6419 6421, 1985

10) Williams, R. M.; R. W. Armstrong \& J.-S. Dung: Synthesis and antimicrobial evaluation of bicyclomycin analogues. J. Med. Chem. 28: 733 740, 1985

11) Höltje, J. V.; D. Mirelman, N. Sharon \& U. Schwarz: Novel type of murein transglycosylase in Escherichia coli. J. Bacteriol. 124: $1067 \sim 1076,1975$ 
12) Hash, J. H. \& M. V. Rothlauf: The N,O-diacetylmuramidase of Chalaropsis species. J. Biol. Chem. 242: 5586 5590, 1967

13) Glauner, B. \& U. Schwarz: The analysis of murein composition with high pressure liquid chromatography. In The Target of Penicillin. Ed., R. HaKenBeck et al., pp. 29 34, W. de Gruyter and Co., Berlin, 1983

14) Pisabarro, A. G.; M. A. De Pedro \& D. Vázquez: Structural modifications in the peptidoglycan of Escherichia coli associated with changes in the state of growth of the culture. J. Bacteriol. 161: 238 242, 1985

15) Pisabarro, A. G.: Estudio de las modificaciones postinsercionales en el peptidoglicano de Escherichia coli. Ph.D. Thesis. Univ. Autónoma of Madrid, 1985

16) Braun, V. \& V. Bosch: Repetitive sequences in the murein-lipoprotein of the cell wall of Escherichia coli. Proc. Natl. Acad. Sci. U.S.A. 69: 970 974, 1972

17) MÜlleR, B. W.; O. ZAK, W. KumP, W. Tosch \& O. WACKer: Semisynthetic bicyclomycin derivatives: Preparation and antibacterial evaluation. J. Antibiotics 32: 689 705, 1979

18) ToKuma, Y.; S. KodA, T. MrYoshi \& Y. MоRiмото: The crystal and molecular structure of bicyclomycin, a new antibiotic. Bull. Chem. Soc. Jpn. 47: 18 23, 1974

19) MaAg, H.; J. F. Blount, D. L. Coffen, T. V. Steppe \& F. Wong: Structure, absolute configuration, and total synthesis of an acid-catalyzed rearrangement product of bicyclomycin. J. Am. Chem. Soc. 100: $6786 \sim 6788,1978$ 